\title{
C-H... H-bonded complexes: How does basis set superposition error change their potential-energy surfaces?
}

\author{
P. Salvador, S. Simon, and M. Duran ${ }^{\mathrm{a})}$ \\ Department of Chemistry and Institute of Computational Chemistry, University of Girona, 17071 Girona, \\ Catalonia, Spain
}

\author{
J. J. Dannenberg \\ Department of Chemistry, City University of New York-Hunter College and The Graduate School, \\ 695 Park Avenue, New York, New York 10021
}

(Received 26 April 2000; accepted 7 July 2000)

\begin{abstract}
Geometries, vibrational frequencies, and interaction energies of the $\mathrm{CNH}^{\cdots} \mathrm{O}_{3}$ and $\mathrm{HCCH} \cdots \mathrm{O}_{3}$ complexes are calculated in a counterpoise-corrected (CP-corrected) potential-energy surface (PES) that corrects for the basis set superposition error (BSSE). Ab initio calculations are performed at the Hartree-Fock (HF) and second-order Møller-Plesset (MP2) levels, using the 6-31G(d,p) and $D 95++(d, p)$ basis sets. Interaction energies are presented including corrections for zero-point vibrational energy (ZPVE) and thermal correction to enthalpy at $298 \mathrm{~K}$. The CP-corrected and conventional PES are compared; the uncorrected PES obtained using the larger basis set including diffuse functions exhibits a double well shape, whereas use of the $6-31 \mathrm{G}(d, p)$ basis set leads to a flat single-well profile. The CP-corrected PES has always a multiple-well shape. In particular, it is shown that the CP-corrected PES using the smaller basis set is qualitatively analogous to that obtained with the larger basis sets, so the $\mathrm{CP}$ method becomes useful to correctly describe large systems, where the use of small basis sets may be necessary. (C) 2000 American Institute of Physics. [S0021-9606(00)30937-0]
\end{abstract}

\section{INTRODUCTION}

Stabilizing interactions between $\mathrm{C}-\mathrm{H}$ bonds and electronegative atoms (particularly oxygen) are well known features of many crystal structures. Even though these kinds of interactions have typical bond energies of $\sim 2-5 \mathrm{kcal} / \mathrm{mol}$, cooperative effects can play an important role in crystal packing $^{1}$ or molecular conformation. The last years have seen an active discussion on the nature of such interactions; furthermore, their characterization as actual hydrogen bonds has become doubtful (see Refs. 2 and 3 and references therein). However, in a recent paper, Gu et al. ${ }^{2}$ showed that the features of the $\mathrm{C}-\mathrm{H} \cdots \mathrm{O}$ interactions are very analogous to the standard $\mathrm{O}-\mathrm{H} \cdots \mathrm{O}$ hydrogen bond interactions, though they are much weaker as a consequence of the smaller acidity of the proton involved. By comparing a $\mathrm{C}-\mathrm{H}$ hydrogenbonded complex to the water dimer in terms of the energetic contributions to the bonding, these authors found that there is no actual distinction between the two types of hydrogen bonds. In both cases, the main opposite forces operating in the interactions are the electrostatic and the exchange ones. Thus, the blueshift effect observed for some of these complexes is just a consequence of the balance of two sets of forces acting in opposite directions.

In a previous paper, ${ }^{3}$ Turi and Dannenberg studied several complexes containing intermolecular $\mathrm{C}-\mathrm{H} \cdots \mathrm{O}$ interactions. They dealt with complexes between acetylene or hydrogen cyanide (as hydrogen donors) and water, formaldehyde or ozone molecules. For each system, full ge-

${ }^{\text {a)} E l e c t r o n i c ~ m a i l: ~ q u e l @ s t a r k . u d g . e s ~}$ ometry optimizations and harmonic vibrational frequency calculations were carried out. Interaction energies corrected for basis set superposition error (BSSE), ${ }^{4}$ zero-point vibrational energy (ZPVE) and thermal correction to enthalpy at $298 \mathrm{~K}$ were also computed. The calculations were made at the Hartree-Fock and MP2 levels of theory. For the complexes involving ozone, two geometries were found on the potential-energy surface (PES), depending on the basis set used. With the 6-31G $(d, p)$ basis sets, a three-center hydrogen bond between the hydrogen atom of the donor molecule and the oxygen atoms of ozone was predicted. On the contrary, using the larger $D 95++(d, p)$ basis set, nonsymmetric structures were found, where the ozone molecule rotated in the molecular plane so that one hydrogen bond was $\sim 0.5-$ $0.7 \AA$ shorter than the other bond. Further ZPVE, BSSE, and enthalpy corrections stabilized the symmetric structure with respect to the nonsymmetric complex, the largest differences being $\sim 0.1 \mathrm{Kcal} / \mathrm{mol}$.

It has been well known for years that BSSE does not only change the interaction energy estimation, but also that its extent is dependent on the intermolecular geometrical parameters of the complex. ${ }^{5-8}$ Therefore, for a proper location of the stationary points on a corrected PES, the BSSEcorrection term must be taken into account at every step of the optimization procedure. ${ }^{5}$

The most common procedure to correct the single-point interaction energy for BSSE has been the a posteriori counterpoise correction scheme (CP correction) proposed by Boys and Bernardi. ${ }^{9}$ Going one step beyond, in a previous paper $^{5}$ we described a simple algorithm to automate the ge- 
ometry optimization of molecular orbitals calculations of complexes on PES that are corrected for BSSE using the counterpoise method. We showed that changes in the PES translated into modification of interaction energies, optimal geometries and vibrational frequencies.

In order to obtain PES corrected for BSSE, it is necessary to optimize $E_{\text {super }}^{\mathrm{CP}}$, as described in Eq. (1), where $E_{\text {super }}$ represents the total energy of the supermolecular aggregate containing $n$ fragment units. The CP correction is stated in Eq. (2), where, the various $E_{m}$ stand for the energies of the individual fragments. The subscript "opt" and " $f$ " denote the individually optimized geometries and the fragments frozen in their supermolecular geometries, respectively. The asterisk $\left(^{*}\right)$ represents fragments calculated with "ghost" orbitals, i.e., using the whole basis sets of the complex. This correction term can be split into the different contributions of all the fragments composing the supermolecule. The CPcorrected interaction energy calculated at the CP-optimized geometry, $E_{\text {int }}^{\mathrm{CP}}$, is expressed by Eq. (4)

$$
\begin{aligned}
& E_{\text {super }}^{\mathrm{CP}}=E_{\text {super }}+\mathrm{CP}, \\
& \mathrm{CP}=\sum_{i=1}^{n}\left(E_{m f}^{i}-E_{m f}^{i^{*}}\right)=\sum_{i=1}^{n} \Delta E_{i}, \\
& E_{\text {super }}^{\mathrm{CP}}=E_{\text {super }}+\sum_{i=1}^{n} \Delta E_{i}, \\
& E_{\text {int }}^{\mathrm{CP}}=E_{\text {super }}+\sum_{i=1}^{n} \Delta E_{i}-\sum_{i=1}^{n} E_{m \text { opt }}^{i} .
\end{aligned}
$$

The first derivatives of $E_{\text {super }}^{\mathrm{CP}}$ with respect to all geometrical coordinates of the complex (needed to find a geometry stationary point), and second Cartesian derivatives (needed to compute force constants and vibrational frequencies) can be expressed as a simple sum of first and second derivatives of $E_{\text {super }}$ and the energies of each fragment with and without ghost orbitals, as indicated by Eqs. (5) and (6)

$$
\begin{aligned}
& \frac{\partial E_{\text {super }}^{\mathrm{CP}}}{\partial p_{j}}=\frac{\partial E_{\text {super }}}{\partial p_{j}}+\sum_{i=1}^{n}\left(\frac{\partial E_{m f}^{i}}{\partial p_{j}}-\frac{\partial E_{m f}^{i^{*}}}{\partial p_{j}}\right), \\
& \frac{\partial^{2} E_{\text {super }}^{\mathrm{CP}}}{\partial p_{j} \partial p_{k}}=\frac{\partial^{2} E_{\text {super }}}{\partial p_{j} \partial p_{k}}+\sum_{i=1}^{n}\left(\frac{\partial^{2} E_{m f}^{i}}{\partial p_{j} \partial p_{k}}-\frac{\partial^{2} E_{m f}^{i^{*}}}{\partial p_{j} \partial p_{k}}\right) .
\end{aligned}
$$

PES for weakly bound complexes are very flat, so the changes in geometry lead to small changes in electronic interaction energies even for relatively large differences in the bond lengths or angles. The CP correction evaluated on the uncorrected PES minima, i.e., single-point counterpoise correction to the interaction energy (henceforth SP), leads to point labeled $\mathbf{d}$ in the PES of Figs. 1(a) and 1(b). It can be seen that, in general, it does not correspond to the geometry of the CP-corrected surface minimum (point $\mathbf{c}$ ). It is wellknown that, because BSSE carries some artificial binding, BSSE-corrected intermolecular distances are increased (using either the counterpoise method or any aprioristic procedure to eliminate BSSE), whereas intramolecular parameters remain almost unchanged. Besides, the uncorrected and the $\mathrm{CP}$-corrected PES can have different curvature and even to-

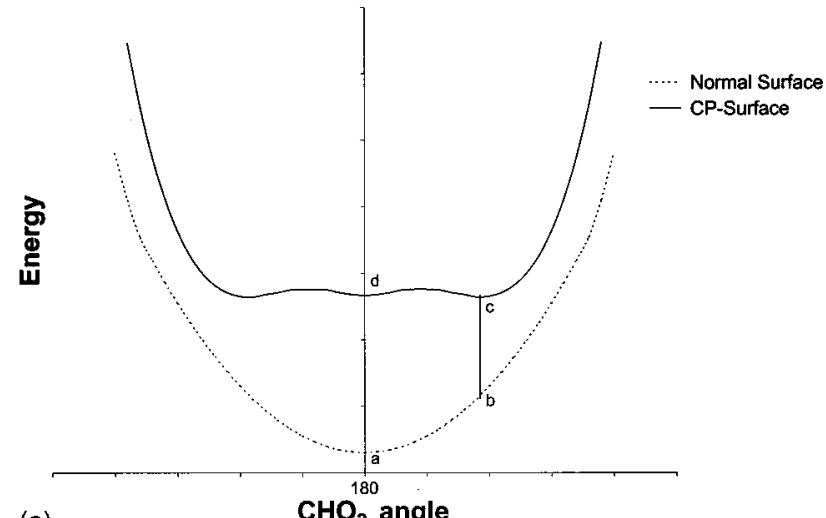

(a)

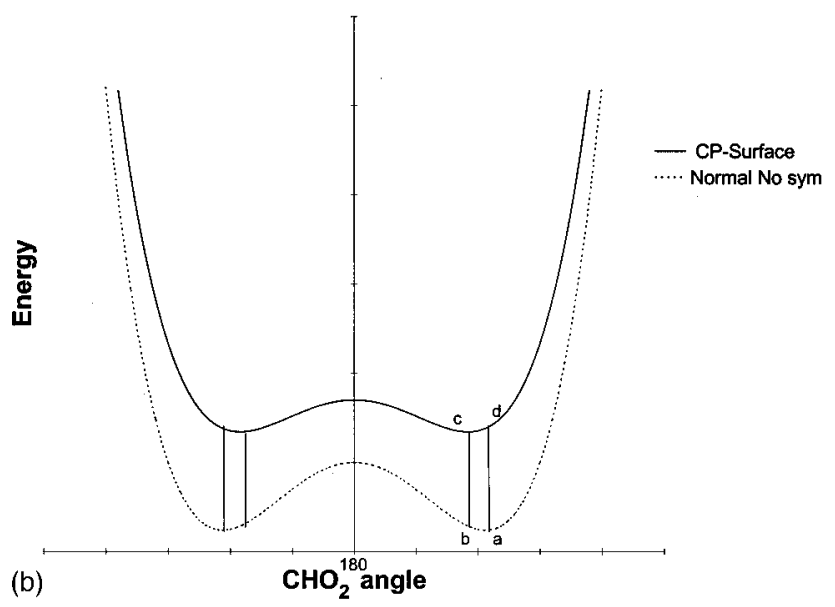

FIG. 1. Comparison between conventional and CP-corrected PES. (a) For the $6-31 \mathrm{G}(d, p)$ basis set. (b) For the $D 95++(d, p)$ basis set. Points $a$ and $c$ mean optimized structures on normal and CP-corrected surfaces, respectively, while points $b$ and $d$ mean optimized geometries on the other surface.

pology. For instance, an earlier work ${ }^{10}$ yielded that the weaker the interaction, the larger the effect BSSE has on the PES of several intermolecular complexes. In that study, for the case of the hydrogen fluoride-water complex for certain basis sets at the DFT and MP2 levels of theory, large differences in the intermolecular arose, although the main internal features of the stationary points remained unchanged. In the same paper, for the well-known water dimer, BSSE contamination distorted the curvature of the PES, by transforming the optimized geometry into a saddle point. Finally, for the hydrogen fluoride dimer, conventional (BSSE-contaminated) optimizations, even with the $6-311 \mathrm{G}(d, p)$ basis set, lead to cyclic structures, whereas the subsequent $\mathrm{CP}$-corrected optimizations yielded the expected linear geometry that are obtained with larger basis sets including diffuse functions.

The aim of this paper is to perform a deeper study of some of the $\mathrm{C}-\mathrm{H} \cdots \mathrm{O}$ complexes studied earlier, ${ }^{3}$ but now taking into account the BSSE effect in both the geometry and the vibrational frequencies. We will assess how the corresponding PES is modified through correction by BSSE, and whether the counterpoise method is still able to yield reliable results using small basis sets. In that case, the CP correction would be a good compromise to obtain accurate descriptions of large systems presenting hydrogen bonds or weak intermo- 
lecular interactions like crystals structures or biological molecules. We have chosen as benchmarks the complexes of cyanide and acetylene with ozone for two reasons. First, the interactions between the donor and acceptor moieties are small enough to foresee that a correct estimation of the BSSE effect is essential to discern between the possible structures the complexes have proved to adopt, namely the symmetric three-center hydrogen bond (single well) and the nonsymmetric bond (double well). Second, because an earlier study by one of us $^{3}$ already suggested that correction for BSSE may transform a double well into a single well. We expect that the deeper analysis of the CP correction by means of a CP-corrected PES will help us to explain under which circumstances a single-well on the PES of some weakly bonded complexes transforms into a double well, depending on the basis set and methodology used, and whether this situation is due to a poor behavior of the method or to a pure BSSE effect.

For this study we chose the counterpoise method for two main reasons: First to compare our results with the previous work, ${ }^{3}$ and second, because the counterpoise method applied at the MP2 level of theory has proved to yield similar results $^{11}$ to the a priori CHA-MP2 BSSE-correction method. Other approaches may exist. For instance, in the last years, the so-called localized second-order Møller-Plesset method $(\mathrm{LMP} 2)^{12}$ have been used for some authors to eliminate to a large extent the BSSE at the correlated level. For instance, Schütz ${ }^{13}$ et al. studied small water clusters at LMP2 level of theory, where they showed that the geometrical parameters and interaction energies obtained with the LMP2 method for the water dimer were comparable to the counterpoisecorrected ones obtained by Xanteas. ${ }^{14}$ The LMP2 method represents a valuable way to reduce the BSSE at the correlated level, although, by construction, it is unable to deal with the BSSE at the HF level, as the localized molecular orbitals are obtained from an uncorrected SCF calculation. Therefore, in case of a large BSSE effect at the uncorrelated level, the LMP2 method might fail to predict reliable results, and a method that eliminates the BSSE at both the uncorrelated and correlated level, like the CHA-MP2 ${ }^{11}$ is required.

The paper is organised as follows. In the next section we will describe the methodology used. Then we will discuss the CP-corrected results obtained in terms of geometries, interaction energies and vibrational frequencies for the complexes, and compare them with both the uncorrected results and the experimental data available. Finally, we will present the conclusions derived from this investigation.

\section{METHOD}

Calculations were carried out at Hartree-Fock ${ }^{15}$ (HF) level and second-order (frozen core) Møller-Plesset ${ }^{16}$ perturbation theory (MP2) levels of theory. Two different basis set were used, namely 6-31G $(d, p)$ and $D 95++(d, p)$. We obtained the CP-corrected PES by writing a short segment of FORTRAN code to drive the energy optimizer, together with several UNIX shell command files. These scripts drove GAUSSIAN $94,{ }^{17}$ the program used to calculate the $2 n+1$ energies, $2 n+1$ derivatives and $2 n+1$ second derivative matrices that were used in the CP correction. The GDIIS ${ }^{18}$
TABLE I. Comparison of the geometric parameters of $\mathrm{CNH} \cdots \mathrm{O}_{3}$ on the normal surface, the $\mathrm{CP}$-optimized surface and the symmetric $\mathrm{CP}$-optimized. Distances in $\AA$ and angles in degrees.

\begin{tabular}{llccccc}
\hline \hline & & \multicolumn{2}{c}{$6-31 \mathrm{G}(d, p)$} & & \multicolumn{2}{c}{$D 95++(d, p)$} \\
\cline { 7 - 7 } \cline { 6 - 7 } & & HF & MP2 & & HF & MP2 \\
\hline$r \mathrm{HO}_{1}$ & Normal & 2.704 & 2.536 & & 3.265 & 2.993 \\
& CP opt & 3.163 & 2.983 & & 3.292 & 3.020 \\
& CP opt (sym.) & 2.771 & 2.631 & & 2.822 & 2.755 \\
$r \mathrm{HO}_{2}$ & Normal & 3.111 & 2.972 & & 3.313 & 3.168 \\
& CP opt & 3.244 & 3.167 & & 3.338 & 3.252 \\
& CP opt (sym.) & 3.185 & 3.108 & & 3.238 & 3.213 \\
$r \mathrm{HO}_{3}$ & Normal & 2.704 & 2.536 & & 2.540 & 2.448 \\
& CP opt & 2.507 & 2.452 & & 2.560 & 2.570 \\
& CP opt (sym.) & 2.771 & 2.631 & & 2.822 & 2.755 \\
$a\left(\mathrm{HO}_{1} \mathrm{O}_{2}\right)$ & Normal & 59.4 & 58.1 & & 77.2 & 70.2 \\
& CP opt & 75.4 & 70.0 & & 77.4 & 68.2 \\
& CP opt (sym.) & 59.3 & 58.1 & & 59.4 & 58.0 \\
$a\left(\mathrm{HO}_{2} \mathrm{O}_{3}\right)$ & Normal & 59.4 & 58.1 & & 41.7 & 45.8 \\
& CP opt & 43.4 & 46.1 & & 41.5 & 47.9 \\
& CP opt (sym.) & 59.3 & 58.1 & & 59.4 & 58.0 \\
$a\left(\mathrm{CHO}_{2}\right)$ & Normal & 180.0 & 180.0 & & 168.6 & 163.7 \\
& CP opt & 168.1 & 167.3 & & 168.2 & 168.2 \\
& CP opt (sym.) & 180.0 & 180.0 & & 180.0 & 180.0 \\
\hline \hline
\end{tabular}

method (direct inversion in the iterative subspace), proposed by Császár and Pulay, was used to ensure a precise localization of the stationary points on the flat surface. The Broyden-Fletcher-Goldfarb-Shanno (BFGS) ${ }^{19}$ variablemetric method to update the inverse Hessian was also used to accelerate convergence. The convergence criterion was set to $10^{-5}$ a.u. in the root-mean-square of the gradient. An initial guess for the inverse Hessian, calculated at the AM1 level of theory, was found to be mandatory for a fast convergence.

For the location of the stationary points on the CPcorrected PES we minimized the CP-corrected supermolecule electronic energy as expressed in Eq. (5). In a second step, vibrational analysis was also carried out for the stationary points of the $\mathrm{CP}$-corrected $\mathrm{PES}$ using the $\mathrm{CP}$-corrected Cartesian second derivative matrix as defined in Eq. (6). As a consequence, both the ZPVE correction and the vibrational contribution to the enthalpic thermal correction term can differ from uncorrected ones, and slightly modify the corresponding interaction energy estimation.

\section{RESULTS AND DISCUSSION}

\section{A. The $\mathrm{CNH} \cdots \mathrm{O}_{3}$ complex}

Results obtained for the $\mathrm{CNH} \cdot \mathrm{O}_{3}$ complex are collected in Tables I-III. Two different planar geometries are considered for this complex. One of them has a symmetric structure, where the $r \mathrm{H}_{1} \mathrm{O}_{1}$ and $r \mathrm{H}_{1} \mathrm{O}_{3}$ distances are equivalent and the $\mathrm{CHO}_{2}$ angle has a value of 180 degrees (see Fig. 2 ). Uncorrected optimizations lead to these symmetric structures with the 6-31G $(d, p)$ basis set, whereas with Dunning's $D 95++(d, p)$ basis set, only nonsymmetric minima are found, which corresponds to the other planar geometry considered.

Uncorrected interaction energies range from -1.51 $\mathrm{Kcal} / \mathrm{mol}$, obtained at the $\mathrm{HF} / / D 95++(d, p)$ level, to the largest interaction energy of $-3.84 \mathrm{Kcal} / \mathrm{mol}$ at the 
TABLE II. Energetic results of the $\mathrm{NCH} \cdots \mathrm{O}_{3}$ complex. The values labeled ZPVE corr. and Enthal. corr. hold for the interaction energy including the ZPVE correction and both the ZPVE and the thermal correction to the enthalpy, respectively.

\begin{tabular}{|c|c|c|c|c|c|c|c|}
\hline \multirow[b]{2}{*}{ Basis set } & \multirow[b]{2}{*}{ Method } & \multirow[b]{2}{*}{ Geom. } & \multicolumn{3}{|c|}{ Interaction energy $(\mathrm{Kcal} / \mathrm{mol})$} & \multirow{2}{*}{$\frac{\Delta E_{\mathrm{O}_{3}}}{(\mathrm{Kca}}$} & \multirow{2}{*}{$\frac{\Delta E_{\mathrm{CNH}}}{\mathrm{mol})}$} \\
\hline & & & Electronic & ZPVE corr & Enthal. corr & & \\
\hline \multirow{8}{*}{$6-31 \mathrm{G}(d, p)$} & $\mathrm{HF}^{\mathrm{a}}$ & Symm. & -2.28 & -1.69 & -1.26 & & \\
\hline & $\mathrm{SP}-\mathrm{HF}^{\mathrm{b}}$ & Symm. & -1.39 & -0.80 & -0.38 & 0.83 & 0.05 \\
\hline & $\mathrm{CP}-\mathrm{HF}^{\mathrm{c}}$ & No Symm. & -1.48 & -0.83 & -0.47 & 0.61 & 0.05 \\
\hline & $\mathrm{CP}_{\mathrm{sym}}-\mathrm{HF}^{\mathrm{d}}$ & Symm. & -1.41 & -0.79 & -0.41 & 0.81 & 0.04 \\
\hline & $\mathrm{MP}^{\mathrm{a}}$ & Symm. & -3.84 & -3.04 & -2.69 & & \\
\hline & SP-MP2 $2^{b}$ & Symm. & -1.66 & -0.86 & -0.51 & 2.03 & 0.14 \\
\hline & $\mathrm{CP}-\mathrm{MP}^{\mathrm{c}}$ & No Symm. & -1.78 & -0.97 & -0.64 & 1.66 & 0.11 \\
\hline & $\mathrm{CP}_{\mathrm{sym}}-\mathrm{MP}^{\mathrm{d}}$ & Symm. & -1.74 & -0.96 & -0.60 & 1.91 & 0.10 \\
\hline \multirow{8}{*}{$D 95++(d, p)$} & $\mathrm{HF}^{\mathrm{e}}$ & No Symm. & -1.51 & -1.01 & -0.56 & & \\
\hline & $\mathrm{SP}-\mathrm{HF}^{\mathrm{f}}$ & No Symm. & -1.35 & -0.85 & -0.41 & 0.09 & 0.05 \\
\hline & $\mathrm{CP}-\mathrm{HF}^{\mathrm{g}}$ & No Symm. & -1.36 & -0.87 & -0.41 & 0.10 & 0.06 \\
\hline & $\mathrm{CP}_{\mathrm{sym}}-\mathrm{HF}^{\mathrm{d}}$ & Symm.(TS) & -1.28 & -0.89 & -0.94 & 0.10 & 0.05 \\
\hline & $\mathrm{MP}^{\mathrm{a}}$ & No Symm. & -2.20 & -1.67 & -1.24 & & \\
\hline & SP-MP2 ${ }^{b}$ & No Symm. & -1.23 & -0.70 & -0.28 & 0.72 & 0.24 \\
\hline & CP-MP2 ${ }^{\mathrm{c}}$ & No Symm. & -1.27 & -0.82 & -0.34 & 0.67 & 0.23 \\
\hline & $\mathrm{CP}_{\mathrm{sym}}-\mathrm{MP}^{\mathrm{d}}$ & Symm.(TS) & -1.26 & -0.88 & -0.93 & 0.64 & 0.23 \\
\hline
\end{tabular}

${ }^{\text {aPoint }} a$ in Fig. 1(a).

${ }^{\mathrm{b}}$ Point $d$ in Fig. 1(a)

${ }^{c}$ Point $c$ in Fig. 1(a).

${ }^{\mathrm{d}}$ Point $c$ in a symmetric surface.

${ }^{\mathrm{e}}$ Point $a$ in Fig. 1(b)

${ }^{\mathrm{f}}$ Point $d$ in Fig. 1(b).

${ }^{\mathrm{g}}$ Point $c$ in Fig. 1(b)

MP2//6-31G $(d, p)$ level of theory. ZPVE and enthalpy corrections decrease the interaction energy by roughly $0.5-0.8$ and $1.0-1.1 \mathrm{Kcal} / \mathrm{mol}$, respectively. Inclusion of correlation energy through the MP2 method increases this value by more than $60 \%$ in all cases.

When the CP correction is added to the uncorrected minima, the numerical dispersion in the different interaction energies clearly decreases. SP-corrected results range from -1.23 to $-1.66,-0.70$ to -0.86 , and -0.28 to $-0.51 \mathrm{Kcal} /$ mol for the electronic, ZPVE-corrected and enthalpycorrected interaction energies, respectively. SP-correction has proved to be larger at the MP2 level for both basis sets; however, the use of diffuse functions clearly decreases its value.

Differing from conventional, BSSE-contaminated results, CP-corrected optimizations yield nonsymmetric minima in all cases. The ozone molecule is displaced farther from the hydrogen and becomes slightly rotated in the molecular plane, so that the intermolecular distances $r \mathrm{HO}_{1}$ and $r \mathrm{HO}_{2}$ increase, whereas $\mathrm{O}_{3}$ approaches the hydrogen atom (see Fig. 2).

Since symmetric structures were located on the uncorrected surface with the 6-31G $(d, p)$ basis set, larger geometric changes appear in this case. Differences of up to $0.4 \AA$ in the intermolecular distances and 10-15 degrees in the hydrogen bond angles are observed upon CP-correction. It is interesting to see that, even though the $r \mathrm{HO}_{1}$ and $r \mathrm{HO}_{2}$ distances clearly increase, the distance to the closest oxygen atom decreases by more than $0.1 \AA$, thus leading to a stronger interaction.

Concerning energetics, $\mathrm{CP}$ and SP electronic interaction energies are reasonably close for both basis sets, despite the large difference in the geometries. This is due to the large flatness of the PES studied. The interaction is so weak that large displacements on the quadratic region of a minimum translate into small energy penalties. However, the SP correction slightly overestimates the effect of BSSE, because it is computed at the uncorrected minimum. Fig. 1(a) depicts the situation in this case: the distance between the points $c$ and $b$ (CP-correction) is always shorter than the distance between $d$ and $a$ (SP-correction).

The behavior of CP-correction changes upon use of diffuse functions; as mentioned above, with the $D 95+$ $+(d, p)$ basis sets, both the conventional and the CPcorrected PES yield nonsymmetric minima. A qualitative graphical representation of both PES is given in Fig. 1(b). At the HF level, the BSSE effect on geometry is very small: Maximum differences of $0.03 \AA$ in the intermolecular distances are observed, whereas the angles remain practically unchanged. At the MP2 level, the CP-corrected intermolecular distances increase by more than $0.1 \AA$ for the $r \mathrm{HO}_{3}$ bond, and the ozone molecule rotates about three degrees. Regarding interaction energies, as both the SP and the $\mathrm{CP}$ corrections are performed on a nonsymmetric surface, the differences are very small. Again, predicted SP-corrected interaction energies are more repulsive than the corresponding $\mathrm{CP}$-corrected ones.

Because all CP-corrected structures are unsymmetrical, we performed $\mathrm{CP}$-corrected optimizations on a symmetryrestricted PES $\left(\mathrm{CP}_{\mathrm{sym}}\right)$ too, i.e., keeping the $r \mathrm{HO}_{1}$ and the $r \mathrm{HO}_{2}$ distances the same, and freezing the angle $a\left(\mathrm{C}_{1} \mathrm{H}_{1} \mathrm{O}_{2}\right)$ to be linear. The nature of the corresponding $\mathrm{CP}_{\text {sym }}$ station- 
TABLE III. Comparison of the vibrational frequencies of $\mathrm{CNH}^{\cdots} \mathrm{O}_{3}$ on the normal surface, the $\mathrm{CP}$-optimized surface and the symmetric $\mathrm{CP}$-optimized surface $\left(\mathrm{cm}^{-1}\right)$

\begin{tabular}{|c|c|c|c|c|c|}
\hline & & \multicolumn{2}{|c|}{$6-31 \mathrm{G}(d, p)$} & \multicolumn{2}{|c|}{$D 95++(d, p)$} \\
\hline & & $\mathrm{HF}$ & MP2 & $\mathrm{HF}$ & MP2 \\
\hline Normal & & 28 & 53 & 24 & 24 \\
\hline CP opt & $\delta$ & 32 & 36 & 25 & 17 \\
\hline CP opt (sym) & & 30 & 43 & $47 i$ & $48 i$ \\
\hline Normal & & 51 & 63 & 71 & 79 \\
\hline CP opt & $\delta_{0}$ & 81 & 94 & 72 & 65 \\
\hline CP opt (sym) & & 58 & 73 & 38 & 31 \\
\hline Normal & & 54 & 67 & 44 & 41 \\
\hline CP opt & $\pi_{1}$ & 65 & 80 & 45 & 42 \\
\hline CP opt (sym) & & 68 & 81 & 42 & 39 \\
\hline Normal & & 64 & 87 & 64 & 66 \\
\hline $\mathrm{CP}$ opt & $\nu$ & 68 & 77 & 61 & 45 \\
\hline CP opt (sym) & & 56 & 67 & 52 & 53 \\
\hline Normal & & 150 & 162 & 121 & 126 \\
\hline CP opt & $\pi_{2}$ & 144 & 160 & 120 & 123 \\
\hline CP opt (sym) & & 156 & 164 & 129 & 124 \\
\hline Normal & & 859 & 737 & 850 & 719 \\
\hline CP opt & & 856 & 735 & 850 & 711 \\
\hline CP opt (sym) & & 860 & 738 & 853 & 698 \\
\hline Normal & & 907 & 772 & 885 & 738 \\
\hline CP opt & & 916 & 791 & 884 & 730 \\
\hline CP opt (sym) & & 908 & 776 & 869 & 726 \\
\hline Normal & & 932 & 845 & 889 & 739 \\
\hline CP opt & & 928 & 833 & 887 & 737 \\
\hline CP opt (sym) & & 930 & 836 & 884 & 736 \\
\hline Normal & & 1447 & 1176 & 1424 & 1185 \\
\hline $\mathrm{CP}$ opt & & 1436 & 1174 & 1424 & 1185 \\
\hline CP opt (sym) & & 1443 & 1176 & 1432 & 1186 \\
\hline Normal & & 1539 & 2041 & 1557 & 1997 \\
\hline $\mathrm{CP}$ opt & & 1548 & 2039 & 1557 & 1997 \\
\hline CP opt (sym) & & 1537 & 2040 & 1546 & 1997 \\
\hline Normal & & 2434 & 2392 & 2405 & 2440 \\
\hline CP opt & & 2433 & 2389 & 2404 & 2440 \\
\hline CP opt (sym) & & 2434 & 2392 & 2405 & 2445 \\
\hline Normal & & 3642 & 3510 & 3619 & 3472 \\
\hline CP opt & & 3633 & 3503 & 3621 & 3477 \\
\hline CP opt (sym) & & 3641 & 3510 & 3627 & 3481 \\
\hline
\end{tabular}

ary point will clarify whether the CP-corrected PES exhibits either a double-well minimum separated by a small energetic barrier, or multiple minima on an extremely flat surface. In the first case, the two equivalent $\mathrm{CP}$-corrected nonsymmetric minima would be connected through a saddle point, which would correspond to the $\mathrm{CP}_{\text {sym }}$. stationary point.

Harmonic frequencies computed at the corresponding stationary points of the three different PES considered (uncorrected, $\mathrm{CP}$ and $\mathrm{CP}_{\text {sym }}$ ) are gathered in Table III. The stationary points of the uncorrected PES are well-defined minima in all cases, showing symmetric and non-symmetric features for the $6-31 \mathrm{G}(d, p)$ and $D 95++(d, p)$ basis sets, respectively. However, when correcting for the BSSE the situation changes. For the smaller basis set, both the symmetric and the nonsymmetric structures located on the $\mathrm{CP}$ and $\mathrm{CP}_{\text {sym }}$ PES, respectively, exhibit real vibrational frequencies, i.e., both are local minima. The energy difference between both geometries represents less than $0.1 \mathrm{kcal} / \mathrm{mol}$ in all cases, and even decreases after ZPVE and enthalpy correction. This difference is smaller than that of the first vibrational level, and hence, the averaged atomic positions correspond to a symmetric structure, which is actually what experimental data ${ }^{20}$ have shown.

On the contrary, for the $\mathrm{CP}_{\text {sym }} D 95++(d, p)$ calculations, the $\mathrm{CP}_{\text {sym }}$ geometries are found to be a saddle point, which must be related to two equivalent $\mathrm{CP}$ nonsymmetric structures. Therefore, one can determine the barrier height to internal rotation, estimated as the energy difference between the $\mathrm{CP}_{\text {sym }}$ and the $\mathrm{CP}$ minima. It is interesting to note that this energy difference becomes negative upon ZPVE or enthalpy corrections, i.e., the symmetric structure is favored respect to the nonsymmetric one. In the latter case, this gap is $\sim 0.5 \mathrm{Kcal} / \mathrm{mol}$. However, this large difference is spurious, as there is one vibrational mode (that assigned to the imaginary frequency) that does not contribute to the enthalpic thermal corrections for the $\mathrm{CP}_{\text {sym }}$ structure, ${ }^{21}$ and thus leads to an artificial lowering in the energy of the $\mathrm{CP}_{\text {sym }}$ interaction energy.

Vibrational analysis shows the presence of up to five intermolecular vibrational modes having an associated vibrational frequency below $200 \mathrm{~cm}^{-1}$. Indeed, such vibrations are those where the $\mathrm{CP}$-correction has a largest effect, not just concerning the vibrational frequency value (the maximum differences observed are $\sim 40 \mathrm{~cm}^{-1}$ ), but especially on the relative ordering of the vibrational modes. We have sorted these intermolecular modes from smaller to larger vibrational frequency, with respect to the values obtained using the $6-31 \mathrm{G}(d, p)$ basis set. These modes are assigned as follows: Modes labeled $\delta$ and $\delta_{0}$ correspond to the oscillation of the $\mathrm{H}$-donor molecule respect to the ozone moiety in the molecular plane (for the $\delta_{0}$ case, the motion of the ozone molecule is practically negligible). Two out-of-plane bending modes are marked as $\pi_{1}$ and $\pi_{2}$, and finally, $\nu$ holds for the $\mathrm{XH} \cdots \mathrm{O}_{3}$ stretching frequency.

The overall effect of BSSE correction is similar to the effect of improving the basis set; for instance, for the the $6-31 \mathrm{G}(d, p)$ basis set, the $\delta_{0}$ frequency is smaller than one of the out-of-plane bendings $\left(\pi_{1}\right)$ and the $\mathrm{CNH} \cdots \mathrm{O}_{3}$ stretching $(\nu)$. Correcting by $\mathrm{CP}$ or improving the basis set both reverse this trend, so their effect is similar.

Regarding the intramolecular modes, the uncorrected and $\mathrm{CP}$-corrected frequencies are very similar, in agreement with previous results for other intermolecular complexes. ${ }^{5}$

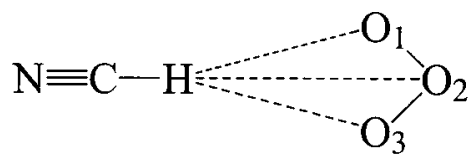

Symmetric

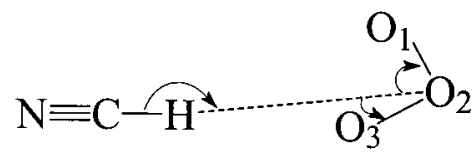

\section{Non-symmetric}

FIG. 2. $\mathrm{CNH} \cdots \mathrm{O}_{3}$ structures. 


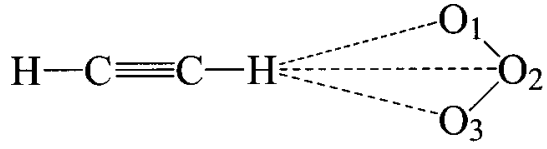

Symmetric

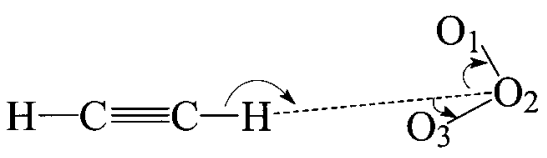

Non-symmetric

FIG. 3. $\mathrm{HCCH} \cdots \mathrm{O}_{3}$ structures.

The comparison of calculated (uncorrected) and experimental vibrational frequencies was already carried out by Turi and Dannenberg. ${ }^{3}$ The same considerations hold after $\mathrm{CP}$-correction, as all experimental frequencies were assigned to intramolecular modes, and the $\mathrm{CP}$ correction has proved to have no meaningful effect on them.

\section{B. The $\mathrm{HCCH} \cdots \mathrm{O}_{3}$ complex}

For this system, two planar geometries have been considered again. First, a symmetric structure presenting two $\mathrm{H} \cdots \mathrm{O}$ interactions, and second a nonsymmetric structure (see Fig. 3). The features of this system turn out to be similar to those of $\mathrm{CNH} \cdots \mathrm{O}_{3}$ complex in terms of geometries and vibrational analysis. Most details discussed above can apply to the acetylene complex. However, the acidic character of the hydrogen atom of the acetylene is not so strong as that of the hydrogen cyanide hydrogen, and thus the intermolecular interaction is weaker. Probably due to this situation, we know of no experimental data for this complex. In practice, we studied the same two planar conformations considered for the $\mathrm{CNH} \cdots \mathrm{O}_{3}$ complex, namely the symmetric and the non-symmetric. The results collected on Tables IV-VI show trends similar to those of the first complex studied. With the 6-31G $(d, p)$ basis set, symmetric stationary points have been located on the uncorrected PES at both the HF and MP2 levels of theory. CP-correction changes this situation and nonsymmetric minima are obtained in all cases. Inclusion of diffuse functions dramatically decreases the BSSE effect on the PES.

Compared to the hydrogen cyanide-ozone complex, the uncorrected intermolecular distances are systematically larger, whereas the interaction energy decreases. However, all different correction terms to the energy, namely the CP and SP, ZPVE and enthalpy corrections, are of the same magnitude, so the corresponding corrected interaction energies are less negative and even in some cases, e.g., the enthalpy-corrected ones, the interaction turns out to be repulsive.

The energetic difference between the symmetric and nonsymmetric structures on the CP-corrected PES is also inverted when the ZPVE correction is included. Larger dif-
TABLE IV. Comparison of the geometric parameters of $\mathrm{HCCH} \cdots \mathrm{O}_{3}$ on the normal surface, the CP-optimized surface and the symmetric CP-optimized. Distances in $\AA$ and angles in degrees.

\begin{tabular}{llccccc}
\hline \hline & & \multicolumn{2}{c}{$6-31 \mathrm{G}(d, p)$} & & \multicolumn{2}{c}{$D 95++(d, p)$} \\
\cline { 7 - 7 } \cline { 6 - 7 } \cline { 6 - 7 } & & HF & MP2 & & HF & MP2 \\
\hline$r \mathrm{H}_{1} \mathrm{O}_{1}$ & Normal & 2.888 & 2.642 & & 3.420 & 3.271 \\
& CP opt & 3.358 & 3.203 & & 3.472 & 3.277 \\
& CP opt (sym.) & 3.002 & 2.819 & & 3.067 & 2.888 \\
$r \mathrm{H}_{1} \mathrm{O}_{2}$ & Normal & 3.307 & 3.086 & & 3.531 & 3.357 \\
& CP opt & 3.474 & 3.507 & & 3.550 & 3.434 \\
& CP opt (sym.) & 3.430 & 3.282 & & 3.499 & 3.357 \\
$r \mathrm{H}_{1} \mathrm{O}_{3}$ & Normal & 2.888 & 2.642 & & 2.794 & 2.529 \\
& CP opt & 2.747 & 2.579 & & 2.782 & 2.659 \\
& CP opt (sym.) & 3.002 & 2.819 & & 3.067 & 2.888 \\
$a\left(\mathrm{H}_{1} \mathrm{O}_{2} \mathrm{O}_{1}\right)$ & Normal & 59.5 & 58.1 & & 74.8 & 75.0 \\
& CP opt & 74.4 & 72.2 & & 76.5 & 72.1 \\
& CP opt (sym.) & 59.4 & 58.1 & & 79.5 & 58.1 \\
$a\left(\mathrm{H}_{1} \mathrm{O}_{2} \mathrm{O}_{3}\right)$ & Normal & 59.5 & 58.1 & & 44.2 & 41.2 \\
& CP opt & 44.5 & 44.0 & & 42.5 & 44.1 \\
& CP opt (sym.) & 59.4 & 58.1 & & 59.5 & 58.1 \\
$a\left(\mathrm{C}_{1} \mathrm{H}_{1} \mathrm{O}_{2}\right)$ & Normal & 180.0 & 180.0 & & 174.5 & 180.0 \\
& CP opt & 171.5 & 168.9 & & 172.5 & 170.9 \\
& CP opt (sym.) & 180.0 & 180.0 & & 180.0 & 180.0 \\
\hline \hline
\end{tabular}

ferences on the enthalpy-corrected energies are found for the $D 95++(d, p)$ calculations for the same reasons brought above for $\mathrm{NCH} \cdots \mathrm{O}_{3}$.

Concerning the vibrational features, it is interesting to see that the frequency labeled $\delta_{0}$, in this case assigned to the oscillation of the $\mathrm{HCCH}$ molecule respect to the ozone, is larger than both $\pi_{1}$ and $\nu$ for the $\mathrm{HF} / 6-31 \mathrm{G}(d, p)$ calculation, contrary to the behavior found in the $\mathrm{NCH}^{\cdots} \mathrm{O}_{3}$ complex.

\section{Single vs multiple well}

One may wonder why and under which circumstances a single well on the PES transforms into a double well (or shallow multiple well) when either BSSE is eliminated at the geometry level or the basis set size is increased. To get a better insight into this point, in the last column of Tables II and $\mathrm{V}$, we collect the energy differences between the fragment calculation with its own basis set and with the basis functions of the whole complex for the ozone and the corresponding donor molecules, labeled $\Delta E_{\mathrm{O}_{3}}$ and $\Delta E_{\mathrm{CNH}}$ or $\Delta E_{\mathrm{HCCH}}$, respectively. The energy differences are calculated at the uncorrected, $\mathrm{CP}$-corrected and $\mathrm{CP}_{\text {sym }}$-corrected stationary points. Note that the sum of both energy differences yields the BSSE value as defined in Eq. (2). The bigger the energy difference, the larger BSSE-like delocalizations are found for the corresponding fragment. From the reported results several conclusions can be drawn; first of all, the most relevant contribution to the BSSE term is caused by the partial delocalizations of the ozone orbitals onto the donor molecule atomic orbitals, mainly due to the proximity of the $s$ and $p$ hydrogen atomic orbitals to the oxygen atoms labeled $\mathrm{O}_{1}$ and $\mathrm{O}_{3}$.

At the MP2 level of theory, the BSSE extent is much more important. In this case, besides the artificial stabilization of the fragment molecular orbitals as a consequence of 
TABLE V. Energetic results of $\mathrm{HCCH} \cdots \mathrm{O}_{3}$ complex. The values labeled ZPVE, corr. and Enthal. corr. hold for the interaction energy including the ZPVE correction and both the ZPVE and the thermal correction to the enthalpy, respectively.

\begin{tabular}{|c|c|c|c|c|c|c|c|}
\hline \multirow[b]{2}{*}{ Basis set } & \multirow[b]{2}{*}{ Method } & \multirow[b]{2}{*}{ Geom. } & \multicolumn{3}{|c|}{ Interaction energy $(\mathrm{Kcal} / \mathrm{mol})$} & $\Delta E_{\mathrm{O}_{3}}$ & $\Delta E_{\mathrm{HCCH}}$ \\
\hline & & & Electronic & ZPVE corr & Enthal. corr & \multicolumn{2}{|c|}{$(\mathrm{Kcal} / \mathrm{mol})$} \\
\hline \multirow{8}{*}{$6-31 \mathrm{G}(d, p)$} & $\mathrm{HF}^{\mathrm{a}}$ & Symm. & -1.37 & -0.82 & -0.34 & & \\
\hline & $\mathrm{SP}-\mathrm{HF}^{\mathrm{b}}$ & Symm. & -0.55 & 0.00 & 0.48 & 0.80 & 0.02 \\
\hline & $\mathrm{CP}-\mathrm{HF}^{\mathrm{c}}$ & No Symm. & -0.60 & -0.07 & 0.41 & 0.59 & 0.02 \\
\hline & $\mathrm{CP}_{\mathrm{sym}}-\mathrm{HF}^{\mathrm{d}}$ & Symm. & -0.57 & 0.01 & 0.46 & 0.76 & 0.02 \\
\hline & $\mathrm{MP}^{\mathrm{a}}$ & Symm. & -2.92 & -2.16 & -1.76 & & \\
\hline & SP-MP2 $2^{b}$ & Symm. & -0.83 & -0.07 & 0.33 & 1.98 & 0.11 \\
\hline & $\mathrm{CP}-\mathrm{MP}^{\mathrm{c}}$ & No Symm. & -0.97 & -0.22 & 0.18 & 1.49 & 0.07 \\
\hline & $\mathrm{CP}_{\text {sym }}-\mathrm{MP}^{\mathrm{d}}$ & Symm. & -0.93 & -0.12 & 0.25 & 1.80 & 0.06 \\
\hline \multirow{8}{*}{$D 95++(d, p)$} & $\mathrm{HF}^{\mathrm{e}}$ & No Symm. & -0.82 & -0.39 & 0.09 & & \\
\hline & SP-HF ${ }^{f}$ & No. Symm. & -0.58 & -0.15 & 0.33 & 0.09 & 0.15 \\
\hline & $\mathrm{CP}-\mathrm{HF}^{\mathrm{g}}$ & No. Symm. & -0.58 & -0.15 & 0.33 & 0.09 & 0.14 \\
\hline & $\mathrm{CP}_{\mathrm{sym}}-\mathrm{HF}^{\mathrm{d}}$ & Symm. (TS) & -0.55 & -0.18 & -0.23 & 0.10 & 0.16 \\
\hline & $\mathrm{MP} 2^{\mathrm{e}}$ & No Symm. & -1.75 & -1.21 & -0.76 & & \\
\hline & SP-MP2 ${ }^{f}$ & No Symm. & -0.62 & -0.08 & 0.36 & 0.75 & 0.37 \\
\hline & $\mathrm{CP}^{\mathrm{MP}} 2^{\mathrm{g}}$ & No Symm. & -0.67 & -0.21 & 0.30 & 0.69 & 0.35 \\
\hline & $\mathrm{CP}_{\mathrm{sym}}-\mathrm{MP}^{\mathrm{d}}$ & Symm. (TS) & -0.67 & -0.27 & -0.30 & 0.66 & 0.37 \\
\hline
\end{tabular}

${ }^{a}$ Point $a$ in Fig. 1(a).

${ }^{\mathrm{b}}$ Point $d$ in Fig. 1(a)

${ }^{\mathrm{c}}$ Point $c$ in Fig. 1(a).

${ }^{\mathrm{d}}$ Point $c$ in a symmetric surface.

${ }^{\mathrm{e} P o i n t} a$ in Fig. 1(b)

${ }^{\text {fPoint }} d$ in Fig. 1(b)

${ }^{g}$ Point $c$ in Fig. 1(b)

BSSE-like delocalizations, a large number of virtual orbitals contribute to the MP2 correction energy term when the whole basis set is used for the fragment calculations. Comparing the values obtained with the $6-31 \mathrm{G}(d, p)$ and $D 95$ $++(d, p)$ basis sets, the main contribution at the MP2 level of theory is found for the largest basis set.

A closer look at the reported data shows that the $\Delta E_{\mathrm{O}_{3}}$ is strongly dependent on the geometrical structure of the complex when using the 6-31G $(d, p)$ basis set. The energetic stabilization due to BSSE is $\sim 0.2 \mathrm{kcal} / \mathrm{mol}$ smaller for the nonsymmetric structures with respect to the symmetric ones. At the MP2 level of theory, the difference increases up to 0.4 $\mathrm{kcal} / \mathrm{mol}$. However, the ratio between the MP2 and $\mathrm{HF} \Delta E_{\mathrm{O}_{3}}$ values ranges between 2.3 and 2.7 , independently of the geometry complex. When diffuse functions are included in the basis set, the dependence on the geometry is very small, even at the MP2 level. Again, the ratio between the MP2 and HF $\Delta E_{\mathrm{O}_{3}}$ energies is rather constant in all cases. Thus, it seems that the energy stabilization of the ozone molecule on the ghost-orbital calculation at the MP2 level with respect to the HF level is independent of the molecular geometry considered.

All in all, the underlying reason for the different topology of the uncorrected and the CP-corrected PES obtained with the $6-31 \mathrm{G}(d, p)$ basis set is the strong dependence of the $\Delta E_{\mathrm{O}_{3}}$ value on the molecular geometry.

The behavior of the $\Delta E_{\mathrm{O}_{3}}$ can be understood by an analysis of atomic orbital interplay. In the symmetric case, both $\mathrm{O}_{1}$ and $\mathrm{O}_{3}$ atoms are close to the hydrogen atom of the donor, so in the ghost-orbitals calculation of the ozone mol- ecule, the $p$ atomic orbitals of the hydrogen atom play an important role on BSSE-like delocalizations. ${ }^{22}$ However, in the nonsymmetric structure, the $\mathrm{O}_{3}$ atom is closer to the hydrogen atom but $\mathrm{O}_{1}$ is too far to interact with the hydrogen atomic orbitals. The final balance is that the difference on the BSSE term is large enough to compensate for the energy penalty caused by loss of symmetry, due to the flatness of the PES. Therefore, the CP-corrected PES exhibits a second energy minimum, which is not symmetric.

The inclusion of diffuse basis functions prevents this problem indeed. The BSSE extent does not depend on the angular disposition of the fragments, but mainly on the intermolecular $r \mathrm{HO}_{3}$ distance. In this case, both the uncorrected and the $\mathrm{CP}$-corrected show similar topologies, the symmetric structure being saddle-point.

\section{CONCLUSIONS}

The $\mathrm{CP}$ correction has proved to be important at the geometry level for the two complexes studied with basis sets having no diffuse functions. In these cases, the uncorrected optimizations lead only to symmetric structures, whereas the CP-corrected PES exhibit both the symmetric and the nonsymmetric geometries. Despite the large differences in the intermolecular parameters, the CP correction only modifies meaningfully the intermolecular vibrational frequencies, so that the ZPVE and thermal corrections to enthalpy are almost not modified. Numerical dispersion on the reported interaction energies decrease upon $\mathrm{CP}$ correction. SP and CPcorrected interaction energies show very similar values for the complexes studied. For such weakly bonded systems, the 
TABLE VI. Comparison of the vibrational frequencies of $\mathrm{HCCH}^{\cdots} \mathrm{O}_{3}$ on the normal surface, the CP-optimized surface and the symmetric CPoptimized surface $\left(\mathrm{cm}^{-1}\right)$.

\begin{tabular}{|c|c|c|c|c|c|}
\hline & & \multicolumn{2}{|c|}{$6-31 \mathrm{G}(d, p)$} & \multicolumn{2}{|c|}{$D 95++(d, p)$} \\
\hline & & $\mathrm{HF}$ & MP2 & $\mathrm{HF}$ & MP2 \\
\hline Normal & & 33 & 44 & 21 & 17 \\
\hline CP opt & $\delta$ & 27 & 28 & 22 & 8 \\
\hline CP opt (sym) & & 35 & 39 & $24 i$ & $30 i$ \\
\hline Normal & & 41 & 49 & 48 & 49 \\
\hline CP opt & $\pi_{1}$ & 51 & 65 & 43 & 48 \\
\hline CP opt (sym) & & 57 & 68 & 50 & 50 \\
\hline Normal & & 51 & 74 & 42 & 58 \\
\hline CP opt & $\nu$ & 46 & 61 & 47 & 45 \\
\hline CP opt (sym) & & 43 & 54 & 36 & 42 \\
\hline Normal & & 58 & 67 & 57 & 72 \\
\hline CP opt & $\delta_{0}$ & 63 & 78 & 60 & 60 \\
\hline CP opt (sym) & & 61 & 72 & 44 & 32 \\
\hline Normal & & 118 & 130 & 102 & 110 \\
\hline CP opt & $\pi_{2}$ & 113 & 127 & 100 & 108 \\
\hline CP opt (sym) & & 123 & 133 & 104 & 110 \\
\hline Normal & & 817 & 175 & 784 & 541 \\
\hline CP opt & & 816 & 490 & 783 & 537 \\
\hline CP opt (sym) & & 819 & 495 & 781 & 533 \\
\hline Normal & & 821 & 512 & 786 & 542 \\
\hline CP opt & & 818 & 510 & 786 & 538 \\
\hline CP opt (sym) & & 823 & 521 & 786 & 537 \\
\hline Normal & & 855 & 733 & 848 & 731 \\
\hline CP opt & & 854 & 732 & 847 & 731 \\
\hline CP opt (sym) & & 856 & 735 & 849 & 733 \\
\hline Normal & & 896 & 779 & 872 & 747 \\
\hline CP opt & & 896 & 788 & 871 & 743 \\
\hline CP opt (sym) & & 897 & 785 & 866 & 736 \\
\hline Normal & & 910 & 823 & 876 & 750 \\
\hline CP opt & & 904 & 812 & 875 & 746 \\
\hline CP opt (sym) & & 910 & 819 & 874 & 744 \\
\hline Normal & & 1449 & 1174 & 1433 & 1183 \\
\hline CP opt & & 1446 & 1173 & 1433 & 1182 \\
\hline CP opt (sym) & & 1447 & 1173 & 1434 & 1183 \\
\hline Normal & & 1537 & 1997 & 1547 & 1951 \\
\hline CP opt & & 1539 & 1996 & 1547 & 1951 \\
\hline CP opt (sym) & & 1536 & 1996 & 1544 & 1951 \\
\hline Normal & & 2241 & 2394 & 2206 & 2444 \\
\hline CP opt & & 2240 & 2391 & 2205 & 2443 \\
\hline CP opt (sym) & & 2241 & 2394 & 2206 & 2446 \\
\hline Normal & & 3583 & 3496 & 3567 & 3451 \\
\hline CP opt & & 3581 & 3492 & 3568 & 3452 \\
\hline CP opt (sym) & & 3582 & 3494 & 3570 & 3454 \\
\hline Normal & & 3695 & 3584 & 3679 & 3544 \\
\hline CP opt & & 3693 & 3581 & 3680 & 3545 \\
\hline CP opt (sym) & & 3694 & 3583 & 3681 & 3546 \\
\hline
\end{tabular}

$\mathrm{CP}$ correction applied to the geometry is not mandatory when one is interested merely on interaction energies.

However, the BSSE correction term has proved to be strongly dependent on the molecular geometry. The large energy stabilization in the ghost orbital calculations for the ozone molecule at the symmetric arrangement of the complex accounts for the overestimation of the interaction energy at this geometry with respect to a nonsymmetric one, which is hidden by BSSE. When BSSE is taken into account by the $\mathrm{CP}$ correction, both geometric minima are located on the PES, stabilizing slightly the nonsymmetric geometry with respect to the symmetric one.

When diffuse functions are included the situation changes, so both the uncorrected and the CP-corrected PES show the same double-well profile. The BSSE correction term does not depend on the angular features of the complex. In this case, the $\mathrm{CP}_{\text {sym }}$ structures turn out to be saddle points connecting two equivalent nonsymmetric minima. This situation suggests that, using the $6-31 \mathrm{G}(d, p)$ basis set, the CP-correction may not fully account for BSSE; the PES is modified, as nonsymmetric structures are found, but the topology of the symmetric stationary points remains unchanged.

In general, the energy differences between both geometries are so small that the energetic barrier would be very difficult to be observed experimentally. Also, the relative stability could be inverted after inclusion of ZPVE or thermal enthalpy corrections to the interaction energy, although, to be fair, these corrections might require introduction of anharmonicity. We are already working in that direction in our laboratory.

For these complexes, the modification of the geometrical parameters induced by BSSE-correction has proved to be very important when using small basis sets. The most important fact is that the corresponding CP-corrected results reproduce, at least qualitatively, the PES obtained with larger basis including diffuse functions. Thus, the $\mathrm{CP}$ correction involving geometry reoptimizations can be a very useful tool in order to yield good descriptions of larger molecular aggregates (i.e., crystal structures or biomolecules), where the use of large basis sets including diffuse functions is at the moment prohibitive.

\section{ACKNOWLEDGMENTS}

This work has been funded through the Spanish DGES Project No. PB98-0457-C02-01. One of us (P.S.) acknowledges the financial support by CIRIT Grant No. 1998Fl00564.

\footnotetext{
${ }^{1}$ J. Van de Bovenkamp, J. M. Matxain, F. B. van Duijneveldt, and T. Steiner, J. Phys. Chem. 103, 2784 (1999); I. I. Chuev, E. G. Atovmyan, and S. M. Aldoshin, J. Mol. Struct. 474, 177 (1999).

${ }^{2}$ Y. Gu, T. Kar, and S. Scheiner, J. Am. Chem. Soc. 121, 9411 (1999).

${ }^{3}$ L. Turi and J. J. Dannenberg, J. Phys. Chem. 97, 7899 (1993).

${ }^{4}$ H. B. Jansen and P. Ros, Chem. Phys. Lett. 3, 140 (1969).

${ }^{5}$ S. Simon, M. Duran, and J. J. Dannenberg, J. Chem. Phys. 105, 11024 (1996).

${ }^{6}$ S. Simon, M. Duran, and J. J. Dannenberg, J. Phys. Chem. A 103, 1640 (1999)

${ }^{7}$ S. S. Xantheas, J. Chem. Phys. 104, 8821 (1996).

${ }^{8}$ J. Sadlej and P. Mazurek, J. Mol. Struct. 337, 129 (1995).

${ }^{9}$ S. F. Boys and F. Bernardi, Mol. Phys. 19, 553 (1970).

${ }^{10}$ P. Salvador, B. Paizs, M. Duran, and S. Suhai, J. Comp. Chem. (to be published).

${ }^{11}$ I. Mayer and P. Valiron, J. Chem. Phys. 109, 3360 (1998).

${ }^{12}$ A. El Azhary, G. Rauhut, P. Pulay, and H.-J. Werner, J. Chem. Phys. 108, 5185 (1998)

${ }^{13}$ M. Schütz, G. Rauhut, and H.-J. Werner, J. Phys. Chem. A 102, 5997 (1998).

${ }^{14}$ S. S. Xanteas, J. Chem. Phys. 104, 8821 (1996).

${ }^{15}$ C. C. J. Roothaan, Rev. Mod. Phys. 23, 69 (1951).
} 
${ }^{16}$ C. Mbller and M. S. Plesset, Phys. Rev. 46, 618 (1934).

${ }^{17}$ Gaussian 94, M. J. Frisch, G. W. Trucks, H. B. Schlegel et al. (Gaussian, Inc., Pittsburgh PA, 1995).

${ }^{18}$ P. Csázár and P. Pulay, J. Mol. Struct. 114, 31 (1984).

${ }^{19}$ R. Fletcher, Practical Methods of Optimization, Vol. 1 (Wiley, New York, 1980).
${ }^{20}$ Z. Mielke and A. Andrews, J. Phys. Chem. 94, 3519 (1990).

${ }^{21}$ Each vibrational mode contributes with $0.6 \mathrm{kcal} / \mathrm{mol}(\mathrm{kT})$ to the thermal correction to the enthalpy.

${ }^{22}$ The use of the 6-31G $(d)$ basis would probably decrease the BSSE extent for this system. However, the lack of $p$ orbitals on the hydrogen would also rebound on the correct description of the whole complex. 